\title{
PHILOSOPHICAL TRANSACTIONS

\section{Local investigation of the optical properties of subwavelength rectangular holes with a focused beam of electrons}

J. C. Prangsma, D. van Oosten and L. Kuipers

Phil. Trans. R. Soc. A 2011 369, 3456-3471

doi: 10.1098/rsta.2011.0173

\section{References}

\section{Subject collections}

\section{Email alerting service}

This article cites 25 articles

http://rsta.royalsocietypublishing.org/content/369/1950/3456.ful I.html\#ref-list-1

\section{Article cited in:}

http://rsta.royalsocietypublishing.org/content/369/1950/3456.full.html\# related-urls

Articles on similar topics can be found in the following collections

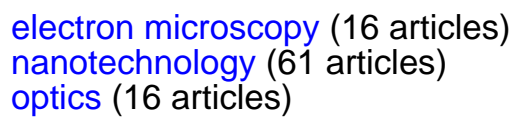

Receive free email alerts when new articles cite this article - sign up in the box at the top right-hand corner of the article or click here

To subscribe to Phil. Trans. R. Soc. A go to:

http://rsta.royalsocietypublishing.org/subscriptions 


\begin{tabular}{l} 
PHILOSOPHICAL \\
TRANSACTIONS \\
$\begin{array}{l}\text { THE ROYAL Phil. Trans. R. Soc. A }(2011) \mathbf{3 6 9}, 3456-3471 \\
\text { SOCIETY }\end{array}$ \\
\hline
\end{tabular}

\title{
Local investigation of the optical properties of subwavelength rectangular holes with a focused beam of electrons
}

\author{
By J. C. Prangsma*, D. van Oosten and L. Kuipers \\ FOM Institute for Atomic and Molecular Physics (AMOLF), Science Park \\ 104, 1098 XG, Amsterdam, The Netherlands
}

\begin{abstract}
The optical properties of rectangular subwavelength holes in a gold film are investigated using the light generated when a focused beam of electrons impinges on the sample close to the hole. Using this technique, multi-spectral maps of the holes are obtained with a resolution beyond the optical diffraction limit. The results show the influence of hole shape on the spectrum of locally scattered light. Rectangular holes of varying shape and size are investigated, and the spatial distribution of the polarization of the observed light is measured. The influence of neighbouring holes is investigated by measuring small clusters of holes.
\end{abstract}

Keywords: transition radiation; extraordinary transmission; surface plasmons;

cathodoluminescence

\section{Introduction}

Thin metallic films perforated by subwavelength apertures have played an important role in the nano-plasmonic research of the past decade $[1,2]$. The large confinement and concomitant field enhancement of light that was necessary to explain the large transmission through hole arrays, as observed in the original experiment came as a large surprise, certainly as up to that point, the transmission through subwavelength holes had been considered a very inefficient process [3]. Soon after the discovery of the remarkable transmission properties of hole arrays, the important role of hole shape became apparent in several investigations [4-6]. This made it all the more clear that the role of the hole as a scatterer and a transmission channel for the light was not well understood. However, with far-field techniques used in most studies, it is not possible to spatially resolve how the shape of the holes influences the optical properties of a structure. Several investigations using near-field scanning probe microscopy have been performed $[7,8]$ without resolving the local effects of hole shape. Although, in the terahertz regime, ingenious pump probe techniques were able to resolve local electric fields with a resolution below the diffraction limit [9], in the optical regime, this has not been achieved yet.

*Author for correspondence (jordprangsma@gmail.com).

One contribution of 9 to a Theo Murphy Meeting Issue 'Metallic metamaterials and plasmonics'. 
Recently, several techniques that use electron beams to obtain optical information beyond the diffraction limit have emerged [10], for example, photon electron emission microscopy (PEEM) [11,12], electron energy-loss microscopy (EELS) $[13,14]$ and electron beam-induced light emission $[4,15-17]$. In this last technique, a focused beam of accelerated electrons impinges on the surface of a sample. The electrons moving through the interface between the vacuum and the sample generate a characteristic electromagnetic radiation spectrum called transition radiation, which is a reason to name this technique transition radiation microscopy. Note that several different names such as cathodoluminescence are used in the literature. This process can be considered to arise from the instantaneous change of the refractive index around the electron as it passes through the interface [18]. Although other mechanisms for the light generation, such as brehmsstrahlung or luminescence are also possible, transition radiation is the dominant contribution for metals at optical frequencies for moderate electron energies [10]. By raster scanning the electron beam over the sample, detailed local optical information can be acquired.

In this paper, we present results using transition radiation microscopy on rectangular holes, elucidating the role of hole shape on the optical properties of the holes. Additionally, we describe polarization-resolved measurements and measurements on small clusters of holes to investigate the role of nearestneighbour interactions.

\section{Experimental technique}

The set-up used for our measurements is an FEI XL30 SFEG scanning electron microscope (SEM) equipped with a cathodoluminescence system by Gatan (figure 1a). A beam of electrons $(30 \mathrm{keV}, 40 \mathrm{nA}$ ) is focused on the sample with a beam waist of $20 \mathrm{~nm}$. The light generated on the sample within a $20 \mu \mathrm{m}$ large area is collected with a parabolic mirror placed under the pole piece of the SEM covering $1.4 \pi$ steradians. In the parabolic mirror, there is a hole with a diameter of $1 \mathrm{~mm}$ to enable the electron beam to pass through to the sample. The collected light is directed towards a spectrometer equipped with a sensitive charge-coupled device (CCD) (Princeton Instruments Spec10 : 100 front illuminated liquid nitrogen cooled). An 'image' is formed by raster scanning the focused electron beam over the sample and collecting a spectrum at each position.

Care must be taken in interpreting the obtained map, as the exact position where the light is emitted is not known owing to the relatively large spot from which the light is collected. The high resolution is thus achieved by using a local 'excitation' of the sample rather than a local detection. The interpretation of the images can be done following the mechanism proposed in Yamamoto \& Suzuki [19] and Kuttge et al. [20], which is summarized here. Two contributions to transition radiation can be distinguished. Firstly, light is directly radiated by the electron crossing the interface. Secondly, the electron generates a surface wave. On a perfectly smooth surface, this surface wave would not reach the detector. However, when scatterers are present on the interface, these can scatter the surface waves into free-space radiation. The two contributions to the generated light are collected with a parabolic mirror and directed towards a spectrometer. The measured light is therefore the interference between the light generated 
(a)

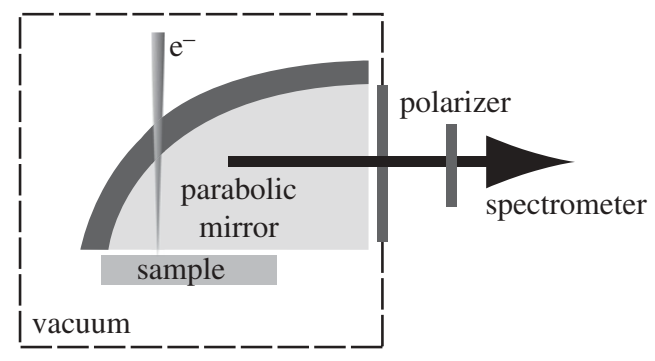

(b)

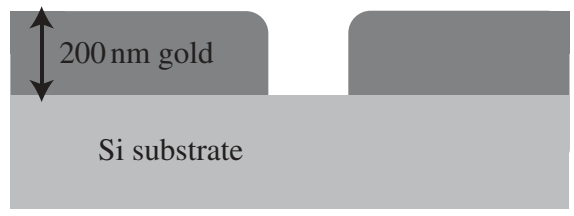

Figure 1. (a) Schematic of the set-up for the detection of electron beam-induced light. In the vacuum chamber of the SEM, the sample is hit by a focused beam of electrons. The light generated is collected with a parabolic mirror and is directed through a vacuum window to a spectrometer. (b) Schematic of the system under investigation showing a subwavelength hole in a $200 \mathrm{~nm}$ thick layer of gold on a silicon substrate.

directly by the electron beam and the light generated by the scattering of surface waves. In the scattering from the small structures that we will discuss in this paper, an additional contribution becomes important: the near-field components of the electron moving through the interface can scatter from the sharp edges of the structure. It is this component that enables highly localized scattering processes and thus allows for the study of the optical properties of the sample on a length scale comparable to the waist of the electron beam.

The structures under investigation in this paper are rectangular holes fabricated with focused ion beam (FIB) milling in a $200 \mathrm{~nm}$ thick layer of gold on a silicon substrate (figure $1 b$ ). The actual dimensions of these small holes are difficult to determine. Scanning electron micrographs show that the holes are slightly tapered and that the edges of the holes have a smooth curvature, as illustrated in figure $1 b$. This was confirmed by atomic force microscope measurements. Owing to the taper and the radius of curvature, it is hard to define the location of the edge from atomic force microscopy data. Therefore, the dimensions of the holes as quoted throughout this paper are the original design parameters. The deviation from design parameters is $10-25 \mathrm{~nm}$, and is caused mainly by the spot size of the FIB. Since these deviations are small, the design parameters give an accurate description of the hole size.

\section{Transition radiation microscopy on a single rectangular hole}

A transition radiation microscopy measurement on a rectangular hole with dimensions $100 \times 260 \mathrm{~nm}^{2}$ is presented in figure 2 . Figure $2 a$ depicts the intensity of collected light while raster scanning the electron beam over the sample. In this image, the colour indicates the total number of integrated counts collected by the detector for each position of the electron beam. Two cross sections of this image are depicted in figure $2 d$. Both cross sections run through the centre of the hole, one along its short axis and one along its long axis. Simultaneously with the optical signal, an SEM signal is collected, which is depicted in figure $2 c$. In the SEM image, three positions are indicated by the letters A, B and C. 
(a)

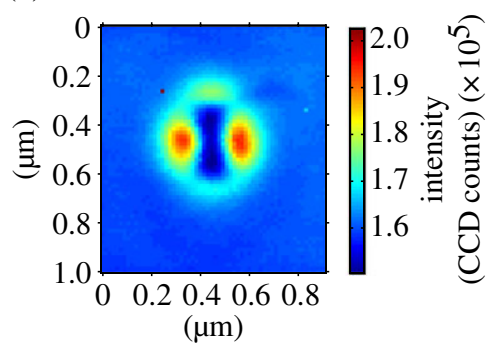

(d)

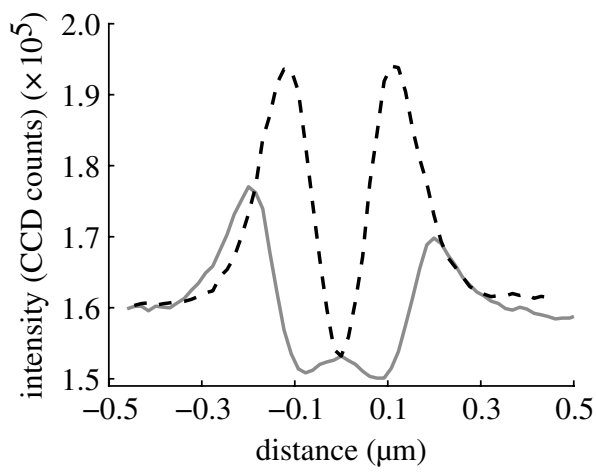

(b)

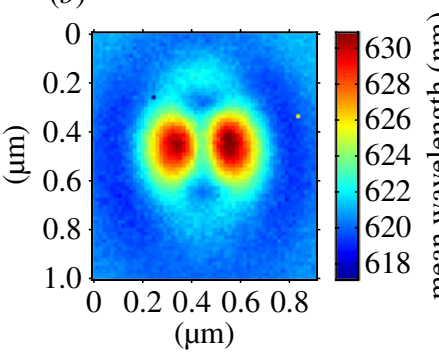

(c)

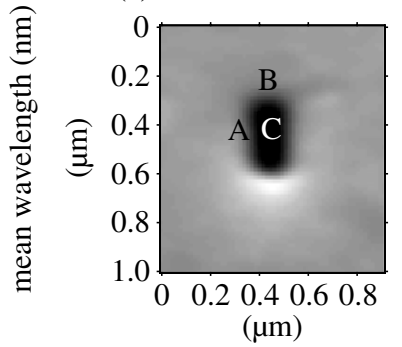

(e)

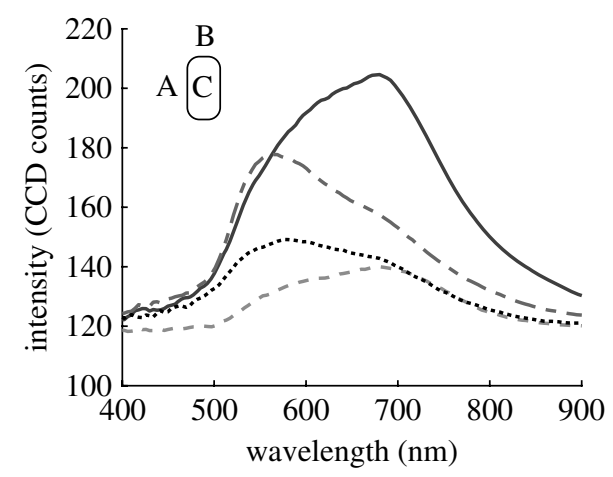

Figure 2. (a) Intensity map of a rectangular hole of $100 \times 260 \mathrm{~nm}^{2}$. The total intensity collected by the spectrometer is presented, i.e. the intensities for all wavelengths have been summed. Clear maxima are visible along the long and short sides of the hole. ( $b$ ) Map of the average wavelength collected on a rectangular hole of $100 \times 260 \mathrm{~nm}^{2}$. This map is constructed from the measured data by calculating the weighted average of the spectrum at each position. The average wavelength yields good qualitative insight into the observed spectra. The spectra along the long side are clearly red shifted with respect to the spectra collected further from the hole. (c) SEM image acquired simultaneously with the intensity map in $(a)$. A, B and $\mathrm{C}$ indicate the positions where the spectra, as shown in $(e)$, were collected. $(d)$ Cross sections of figure 2. Intensity of transition radiation along two lines through the centre of the rectangular hole. The dashed and drawn lines indicate cross sections along the short and long axes, respectively. (e) Four spectra acquired at different positions on a rectangular hole. (A) Spectrum at position A at the peak in intensity at the long side of the hole. (B) Spectrum at the peak in intensity at the top side of the hole. (C) Spectrum at the centre of the hole. (Reference) Spectrum corresponds to the background transition radiation obtained far away from the hole. $(d)$ Grey solid line, vertical cross section; dashed line, horizontal cross section. (e) Grey solid line, A; grey dashed line, B; grey dashed-dotted line, C; black dashed line, reference. (Online version in colour.)

Spectra collected at these positions are presented in figure $2 e$. Figure $2 b$ shows the average wavelength at every pixel position. These data are constructed from the spectra collected at every pixel position by taking the weighted average of the corresponding spectrum. Because the spectra in these measurements always show only one maximum, an image of the average spectrum gives a good qualitative insight into the measured spectra. Figure 3 shows the same dataset integrated over nine consecutive wavelength ranges of $55 \mathrm{~nm}$. 

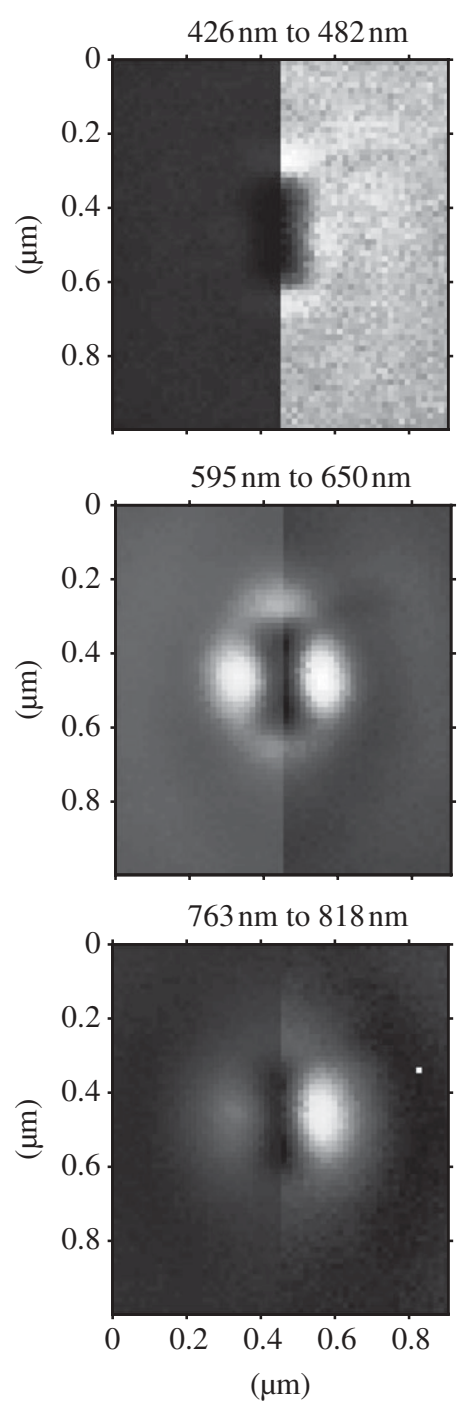

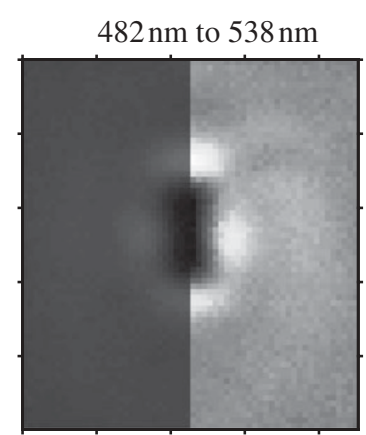

$651 \mathrm{~nm}$ to $706 \mathrm{~nm}$

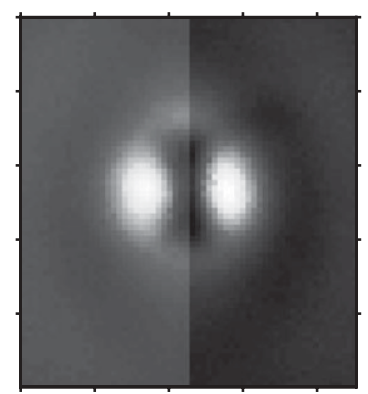

$819 \mathrm{~nm}$ to $874 \mathrm{~nm}$

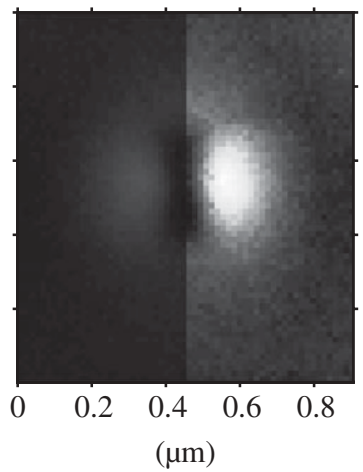

$539 \mathrm{~nm}$ to $594 \mathrm{~nm}$

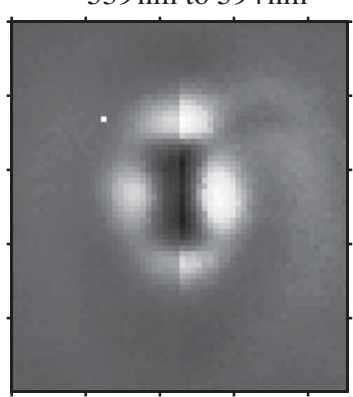

$707 \mathrm{~nm}$ to $762 \mathrm{~nm}$

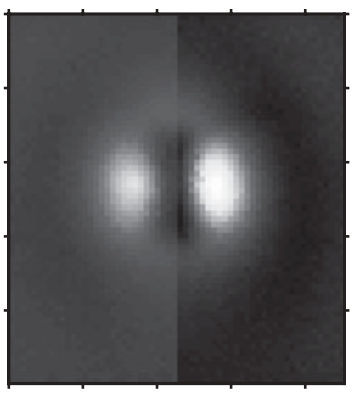

$875 \mathrm{~nm}$ to $930 \mathrm{~nm}$

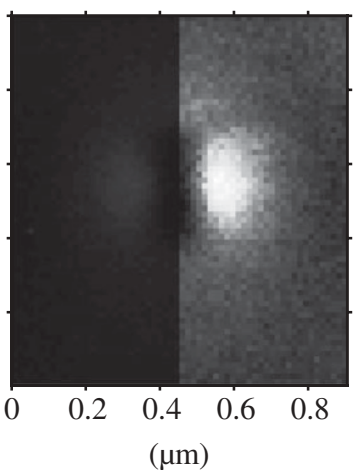

Figure 3. Nine figures of the same dataset as figure 2. Each figure is integrated over an equal wavelength range of $55 \mathrm{~nm}$, the exact range is indicated above the figure. The left-hand side of each figure is scaled to the minimum and maximum of the whole dataset, while the right-hand side of each figure is scaled to the minimum and maximum within the integrated wavelength range. The left-hand sides of the images enable a fair comparison of the measured intensities between different images.

In the measurements, the most prominent features are the two large maxima at the long side of the hole (figure $2 a$ ). At these positions, the signal collected is 25 per cent higher than the signal collected on a plain gold surface. The spatial extent of these maxima is very small; the full-width half maximum of these peaks is $120 \mathrm{~nm}$ (figure $2 d$ ). Apart from the two maxima along the long side, two smaller maxima along the short side of the hole are visible in the intensity map. These two maxima have a difference in intensity that, based on 


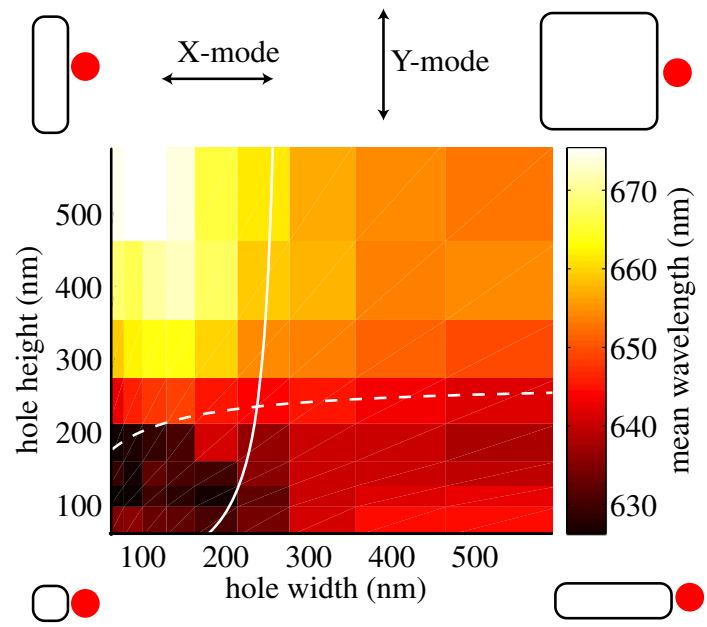

Figure 4. Measured average wavelength as a function of the hole dimensions. The schematic of the hole illustrates how the size of the hole varies throughout the figure, the dot indicates the position where the spectrum was measured. The two white lines indicate the cutoff for $650 \mathrm{~nm}$ for the X-mode (horizontal cutoff line) and Y-mode (vertical cutoff line). (Online version in colour.)

the symmetry of the hole, is unexpected. We attribute this small asymmetry to an angle-dependent collection efficiency of the parabolic mirror along one axis. In figure 3 , the data are integrated over nine consecutive wavelength ranges. It can be seen that the maxima of the short side of the hole attain their peak values at shorter wavelengths then the maxima at the long sides.

The spectra at the positions of the intensity maxima of the long side (position $\mathrm{A}$ ) and top side (position $\mathrm{B}$ ) are shown in figure $2 e$, together with a spectrum collected on a plain gold surface and a spectrum collected inside the hole (position C). Clearly, there is a large difference between the spectra collected at these different positions. The spectrum along the long edge has a peak around $680 \mathrm{~nm}$, while the spectrum at the top side peaks at $560 \mathrm{~nm}$. All spectra measured have only a single maximum. In figure $2 b$, it can be seen that around the long edges, a red shift with respect to the spectrum far from the hole is visible. At the top and bottom peaks along the short side of the hole, the spectrum is blue shifted. Although all the spectra show one peak, it is also clear that the spectra all show more- or less-pronounced shoulders. It is thus possible that the spectra arise from the combination of two or more single-peaked spectra of which the relative intensities change for each position.

The results discussed for this particular hole are very general for all small (less than $500 \mathrm{~nm}$ ) rectangular holes. All holes show single-peaked maxima that are more intense as the side of the rectangle is longer (not shown here). The high peaks at the side of the hole are reminiscent of the high fields that are found on the sides of the hole in theoretical calculations [21]. Since the spectra at the long and short sides of a rectangular hole differ considerably, it is likely that these peaks and their spectra are related with the orthogonal waveguide modes of a rectangular hole. 


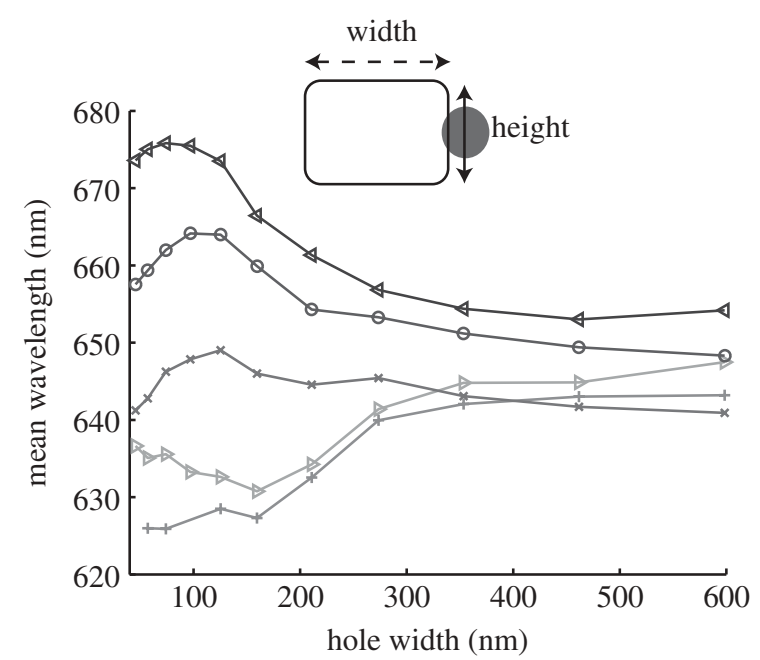

Figure 5. Average wavelength of the spectrum collected at the side of the hole as a function of the hole width, for different hole heights. In the five curves, the hole height is $60 \mathrm{~nm}$ (line with right pointed triangles), $100 \mathrm{~nm}$ (line with vertical bars), $210 \mathrm{~nm}$ (line with crosses), $270 \mathrm{~nm}$ (line with circles) and $460 \mathrm{~nm}$ (line with left pointed triangles), respectively. Above a width of $300 \mathrm{~nm}$, the average wavelength shows little dependence on the width of the hole. Below $300 \mathrm{~nm}$, the width of the hole has a large influence on the average wavelength: below a hole height of $200 \mathrm{~nm}$, there is a decrease in average wavelength, while for holes with larger height, a decrease in width leads to a red shift of the average wavelength.

\section{(a) Systematic investigation of the effect of hole shape}

To perform a systematic study of the role of hole shape, a sample was made in which the width and the height of holes were varied between 50 and $600 \mathrm{~nm}$. For more than 100 holes with different dimensions, a spectrum was collected at the location next to the hole, as indicated schematically by the dot in figure 4 .

As before, all the observed spectra are single peaked. We thus again use the average wavelength of each spectrum as a qualitative measure of the observed spectrum. Figure 4 depicts the average wavelength as a function of hole width and height. Figure 5 shows the average wavelength at the side of the hole as the width of the hole is changed, i.e. these are crosscuts of figure 4 for various hole heights. Clearly, in the range $300-600 \mathrm{~nm}$, the width of the hole has very little influence on the spectrum collected. Below a width of $300 \mathrm{~nm}$ however, the average wavelength depends strongly on the width, but the way it changes depends on the height of the hole. Below a height of $210 \mathrm{~nm}$, the average wavelength decreases as the width is reduced. Above $210 \mathrm{~nm}$ height, the average wavelength first increases as the width is decreased in the region 300-100 nm. Finally, in the region below $100 \mathrm{~nm}$ width, a small decrease is visible for the three spectra with largest heights.

Scattering light to surface plasmon polaritons (SPPs) and vice versa by subwavelength holes has been essential in explaining extraordinary optical transmission and is well known to be wavelength and shape dependent. Experiments by Baudrion et al. [22] showed that the scattering of light to SPPs 
is considerably more efficient for larger holes than for smaller holes. Moreover, the highest efficiency for SPP launching by the hole was observed at the cutoff wavelength of the fundamental waveguide mode of the hole. Analogously, the scattering of the field of the electron at the interface is expected to depend critically on the geometry and the possible optical modes inside the hole. A very small hole, for which all wavelengths are longer than the cutoff wavelength will scatter less than a hole that has propagating waveguide modes. The two white curves in figure 4 indicate the hole dimensions with a cutoff at $650 \mathrm{~nm}$ calculated using the model that was introduced by Collin et al. [23]. With this behaviour of the cutoff in mind, we propose the following explanation of the observed dependence of the average wavelength on the dimensions of the hole. The transition radiation generated close to the hole is enhanced when a waveguide mode exists in the hole. Thus, an increased scattering near the hole and the spectrum measured close to the hole are influenced by the waveguide modes of the hole.

We now consider figure 4. For the holes on the top right side, most of the wavelengths are longer than the cutoff wavelength, decreasing the width, moving to the top left side of the graph will lead to an increase of the cutoff wavelength. This leads to a larger contribution of the long wavelengths to the spectrum scattered from the hole. Starting on the lower right-hand side, we are already below the cutoff for all wavelengths. Decreasing the width of the hole, we observe a blue shift of the spectrum. This is explained by the fact that a non-resonant subwavelength scatterer will scatter short wavelengths more efficiently than long wavelengths. Therefore, decreasing the size of holes that are already in cutoff will lead to a blue shift in the observed spectrum.

The qualitative reasoning above simplified the explanation to low scattering below cutoff and high scattering above cutoff. These statements can be refined further by realizing that the scattering depends on the specific propagation properties of the waveguide and not solely on it being above or below cutoff. Our approach gives a useful guideline to understand transition radiation microscopy measurements near subwavelength apertures.

\section{(b) Polarization-resolved measurements}

To further elucidate the properties of the rectangular apertures, a polarizer was introduced in the beam path. This way the contributions of the different polarizations can be analysed. Figure 6 shows the result of 16 intensity maps measured of the same hole, rotating the analyser after the parabolic mirror in steps of $22.5^{\circ}$ over $360^{\circ}$. Clearly, the light generated by the electrons impinging near the hole has polarization-dependent properties, as the excitation maps differ distinctively for different orientations of the polarizer. The collected light consists of a linearly polarized part and a part that is not linearly polarized. Generally, the last part consists of an unpolarized part, a circular polarized part and a part that arises from the spatial variation of the polarization within the generated light beam.

Interpretation of these measurements is not straightforward as the polarizationdependent collection properties of the parabolic mirror are not trivial. To illustrate this, we consider a dipole source $\alpha$ in the focal point of the parabolic mirror, with its dipole moment oriented along the axis of the paraboloid (figure 7). 

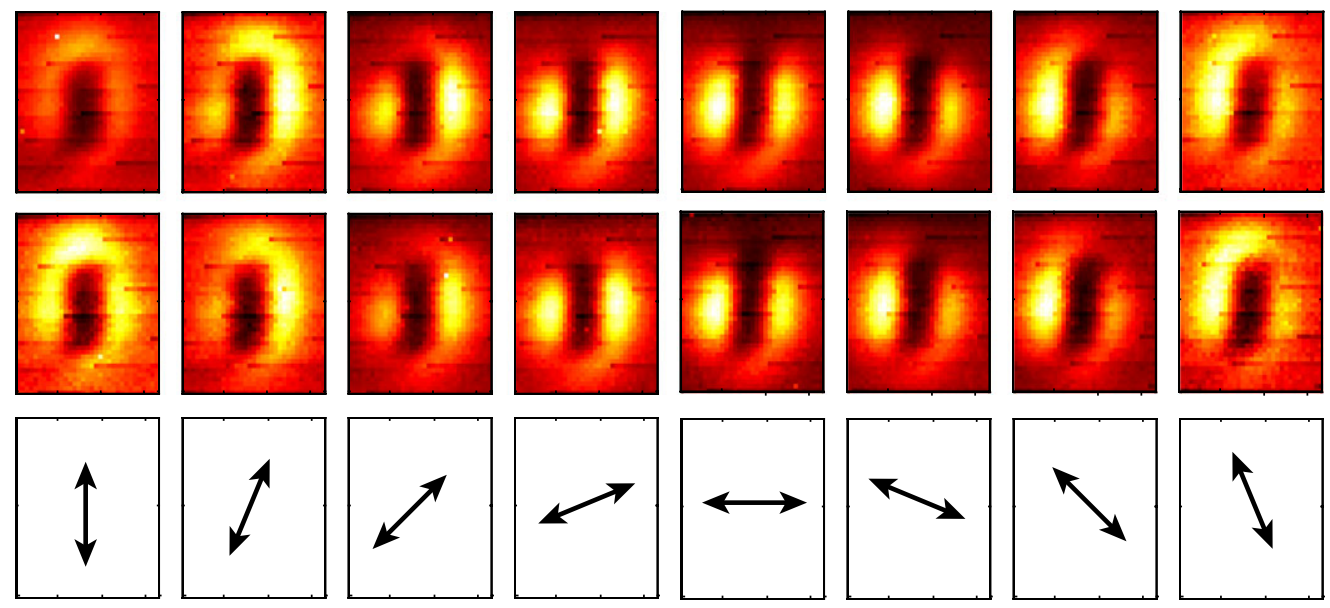

Figure 6. Sixteen intensity maps of the same rectangular hole for 16 settings of the analyser, rotating the polarizer over $2 \pi$ radiants. (Online version in colour.)
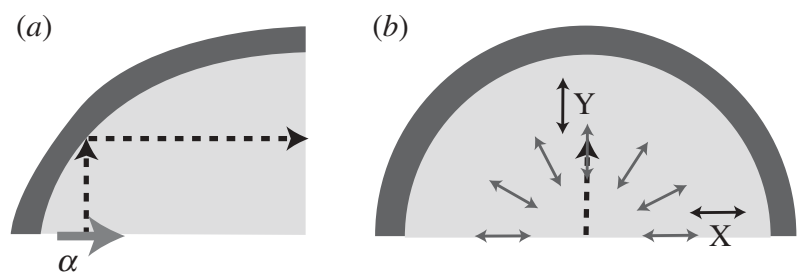

(c)

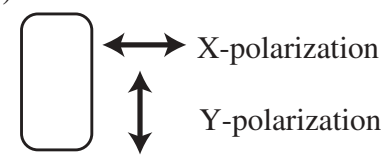

Figure 7. $(a, b)$ Two cross sections of the parabolic mirror. The dashed arrows indicate a light path, the thin arrows indicate polarization directions. A dipole $\alpha$ oriented parallel to the surface, with its dipole moment pointing along the axis of the paraboloid, will emit light that is radially polarized after the mirror. In the interpretation of the measurements presented in this section, it is assumed that most of the scattered light is directed straight up from the surface. This means that the $\mathrm{X}$ - and Y-polarization emitted from the sample indicated schematically by the rectangular hole in $(c)$ are mapped on the $\mathrm{X}$ - and $\mathrm{Y}$-polarization, as indicated in the cross section in $(b)$.

Following the rays from the dipole perpendicular to its dipole moment, the reflected rays form a collimated beam emerging from the mirror. This beam will be radially polarized (figure $7 b$ ). Measuring the intensity of the beam as a function of the angle of the analyser will thus give no clear information on the orientation of the dipole. However, when the light is scattered from the structure in a preferential direction, it is very well possible that information on the polarization is still present. Additionally, when unpolarized light impinges 

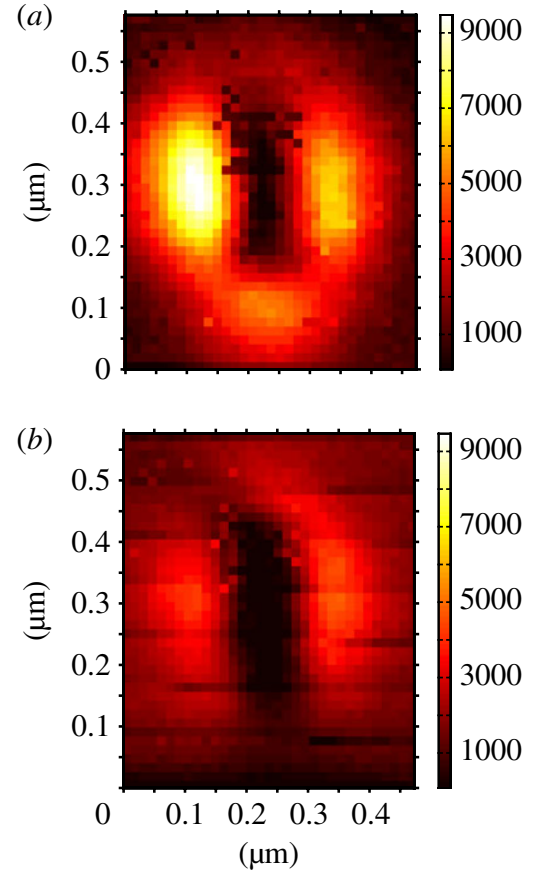

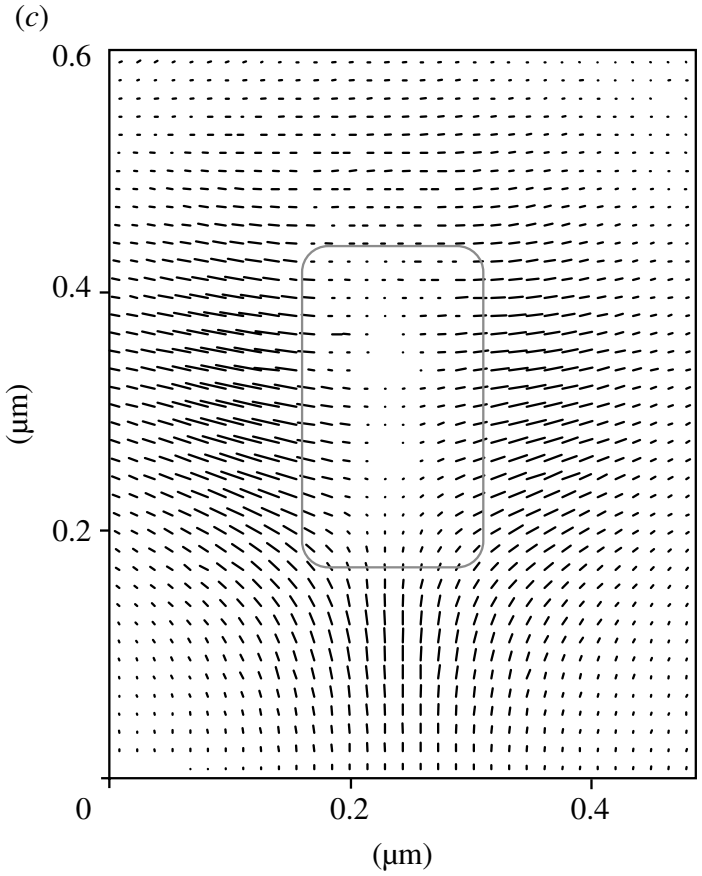

Figure 8. Polarization distribution reconstructed from 16 measurements of a single hole. (a) The intensity map of $I_{\mathrm{p}}$, the amplitude of the linearly polarized contribution. (b) The intensity map of $I_{\mathrm{u}}$, the amplitude of the unpolarized contribution. (c) Spatial map of the magnitude and the orientation of the linearly polarized part $\left(I_{\mathrm{p}}\right)$ indicated by a headless arrow. (Online version in colour.)

on the aluminium parabolic mirror, the reflected light will not become polarized. This means that the polarization dependencies observed in the measurement indicate a polarization-dependent emission.

The above discussion illustrates that care must be taken when interpreting the results. We will assume here that the light scattered at the subwavelength hole is predominantly directed straight up from the surface. In that case, the polarization emitted and collected are related to each other, as indicated by the $\mathrm{X}$ - and Y-polarization drawn in the schematic of figure 7.

For each pixel position, the function $S_{\theta}=I_{\mathrm{u}}+I_{\mathrm{p}}^{*} \cos ^{2}\left(\theta-\theta_{\mathrm{p}}\right)$ is fitted to the measurements, where $S_{\theta}$ is the intensity measured on each pixel for different orientation angles $\theta$ of the analyser. $I_{\mathrm{u}}, I_{\mathrm{p}}$ and $\theta_{\mathrm{p}}$ are fit parameters that correspond to the intensity of the nonlinearly polarized part, the intensity of the linearly polarized part and the orientation of the linearly polarized part, respectively. Figure $8 a, b$ shows the obtained values of $I_{\mathrm{u}}$ and $I_{\mathrm{p}}$ in identical colour scales. Note that close to the hole, the polarized intensity is larger than the unpolarized intensity. In figure $8 c$, the intensity $I_{\mathrm{p}}$ and the angle $\theta_{\mathrm{p}}$ are plotted as headless arrows. The polarization of the emission is oriented perpendicularly to the edges of the hole on the right, left and lower parts of the hole. In the upper part of the hole, the polarization is seemingly oriented parallel to the edge of the hole, but here the linearly polarized contribution is lower in amplitude. The asymmetry that is observed between the top and bottom sides is most probably related to the asymmetric collection efficiency of the parabolic mirror. The observed 
pattern in figure $8 c$ shows that the light is mainly scattered with a polarization perpendicular to the edge. This is in agreement with how one would expect light scattering from an edge to be polarized. The choice made in relating the angle of the polarizer to the polarization of the emitted light thus seems to be correct.

As a last note to the measurements on single holes described in the last two sections, it is interesting to realize that there is a similarity between the radiation of a dipole oriented perpendicular to the surface and the generated transition radiation. This arises from the fact that the main contribution of the radiation is generated when the electron is very close to the surface and the current of the electron can be described as the superposition of many oscillating dipole currents. It was put forward that the transition radiation is a measure of the local density of states (LDOS) on the surface of the metal [20]. The patterns observed in the experiments would thus give a measure of the amount of light that would be collected when a dipole, such as a fluorescent molecule, would be located close to the rectangular hole. Care should be taken in this interpretation, however, since there is no one-on-one relation between the LDOS and the measured signal [24].

\section{Multiple holes}

When an array of subwavelength holes is illuminated by a plane wave, each hole acts as a scatterer at which surface waves are generated. These surface waves can subsequently scatter or reflect at the other holes present in the structure. This scattering process leads to the reflection and transmission of light from the complete structure. In this section, we try to reduce the complexity of the problem by looking at a short chain of three holes. Similar structures have been measured using far-field methods where entire rows of holes were illuminated [25]. In such experiments, all the holes are driven in unison. With transition radiation microscopy, we can locally excite this relatively simple geometry. In the experiment, we will vary the distance between these holes and investigate how this distance influences the spectra measured at several different positions.

In figure 9, transition radiation microscopy intensity images of three configurations of three identical subwavelength holes are shown. In each image, the holes have a width of $260 \mathrm{~nm}$ and a height of $100 \mathrm{~nm}$. The distance $D$ between the holes is different for each arrangement. The excitation patterns observed for arrangements of three holes all appear to be merely the sum of the excitation patterns of the three constituting single holes. In addition, when the images are constructed by integrating narrow ranges in wavelength (image not shown) no clear qualitative changes, that would, for instance, indicate interference effects, are observed.

When the spectra are analysed, it becomes clear that the arrangement of multiple holes is not the same as the sum of single holes. Figure $10 a$ shows the spectra acquired when the electron beam is positioned at the top side of the upper hole of the arrangement (position 1 in the schematic in figure $9 d$ ). The graphs in figure $10 a, c, e$ are for the three arrangements with different spacing. These same data are shown in the graphs of figure 10b,d,f, but here the spectra are normalized to a spectrum collected from a single hole: the plotted value is $\tilde{I}(\lambda)=I_{\text {det }}(\lambda) / I_{\text {ref }}(\lambda)$, with $I_{\text {det }}(\lambda)$ the detected spectrum and $I_{\text {ref }}(\lambda)$ a reference spectrum obtained at the long side of a single isolated hole of the same shape. 
(a)

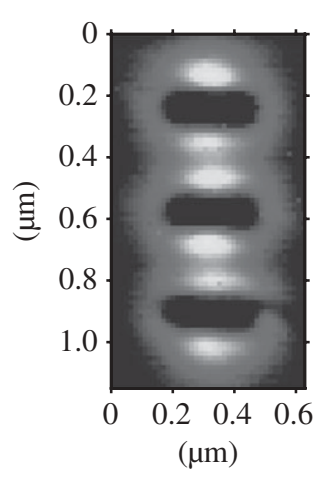

(b)

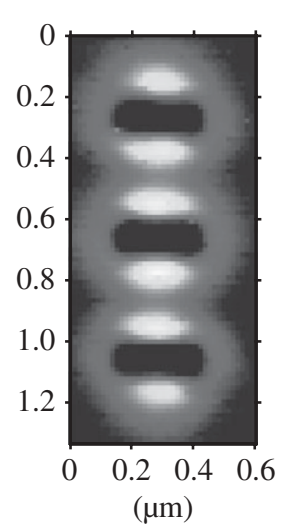

(c)

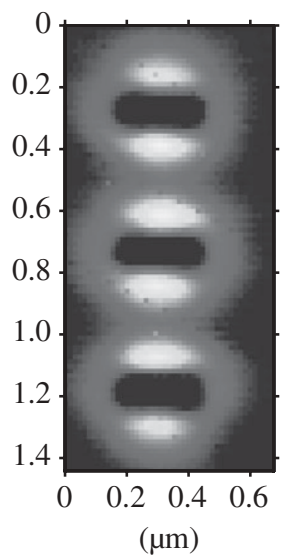

(d)

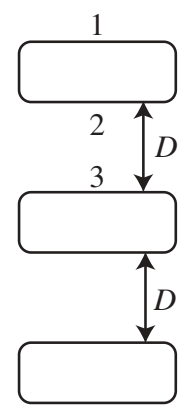

Figure 9. Intensity maps of three configurations of rectangular holes and a schematic overview. The figures $(a-c)$ all have holes of size $100 \times 260 \mathrm{~nm}$. The distance between the holes is $(a) 240,(b) 300$ and $(c) 350 \mathrm{~nm}$. In the schematic $(d)$, the positions 1, 2 and 3 indicate the positions where spectra are shown in figure 10. All three pictures have identical scaling.

The graphs in both figures show the spectra and normalized spectra at positions 1 (figure 10a,b), 2 (figure $10 c, d$ ) and 3 (figure 10e,f) (figure $9 d$ ), respectively. The positions 1,2 and 3 are all located in the high-intensity peaks just above or below the hole.

Clearly, all spectra differ distinctively from the spectra collected at a single hole, all normalized spectra have values mainly above 1 . The intensity of the spectra increases going from position 1 to position 2 to position 3 (figure 10). The amount of light radiated from the structure within the measured range of wavelengths is thus larger than for a single isolated hole. This statement needs some refinement, as it is not true for all wavelengths. The geometry with the smallest spacing gives rise to a small decrease in the spectrum with respect to the single hole, for a small range of wavelengths. In particular, at position 2 (figure 10b), a decrease in the wavelengths around $700 \mathrm{~nm}$ can be observed. Another visible trend in the data is that the peak in both the spectrum and the normalized spectrum shift to the red when the spacing of the holes is increased. The positions of the peaks in the spectra as a function of the hole spacing are plotted in figure 11 .

The observed intensity at every position is generally higher than the intensity measured at a single isolated hole. This behaviour of the intensity is in good correspondence with what one intuitively might expect: more holes lead to more scattering of surface waves and thus to a higher observed intensity. At positions 2 and 3 from a geometric point of view, more scattering is expected than from position 1, as the latter position neighbours a large empty field of gold. Thus, the closer to the centre of the arrangement of the holes, the more scattering will occur.

The fact that the radiated spectrum changes as the spacing between the holes is varied, is an indication that there is an interference effect contributing to the response of the entire structure. Surprisingly, no long-range periodicity is 
(a)

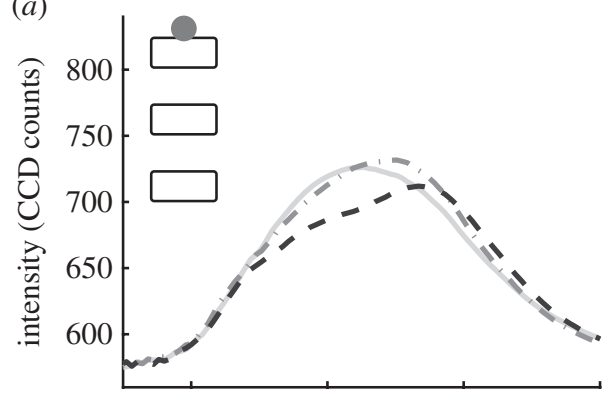

(c)

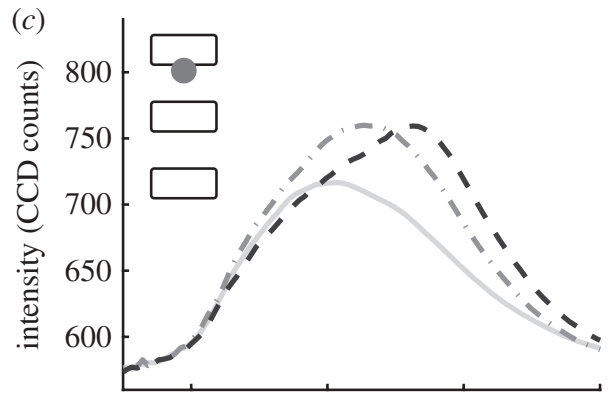

(e)

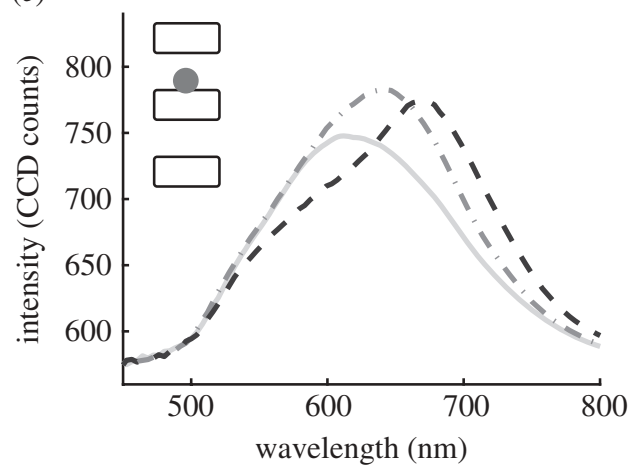

(b)

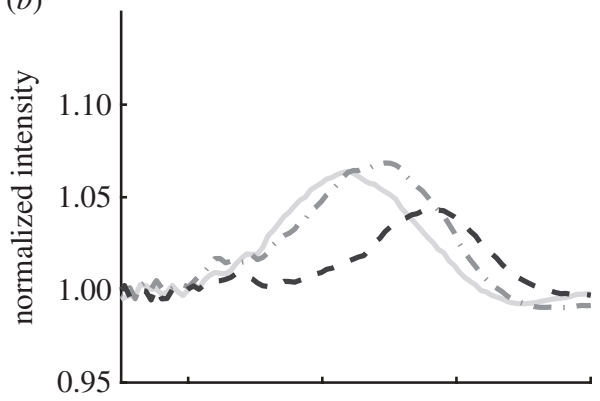

$(d)$

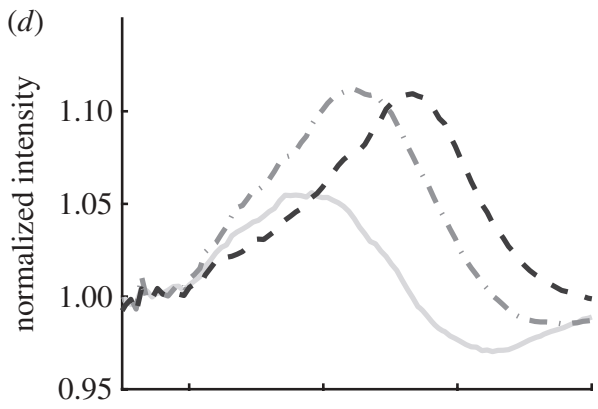

$(f)$

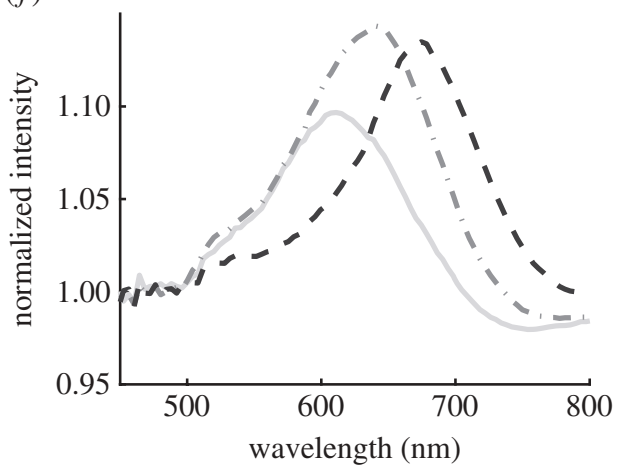

Figure 10. $(a, c, e)$ Spectra at three positions for each position varying the hole spacing and $(b, d, f)$ normalized spectra at three positions for each position varying the hole spacing. ( $a$ ) The spectra at position 1 at the top side of the upper hole. (c) The spectra at position 2 at the bottom side of the top hole. (e) The spectra at the top side of the middle hole. (b) The spectra at position 1 at the top side of the upper hole. $(d)$ The spectra at position 2 at the bottom side of the top hole. (f) The spectra at the top side of the middle hole. The plotted value is $\tilde{I}(\lambda)=I_{\text {det }}(\lambda) / I_{\text {ref }}(\lambda)$, with $I_{\text {det }}(\lambda)$ the detected spectrum and $I_{\text {ref }}(\lambda)$ the spectrum collected at the top maximum of an isolated hole of the same size and shape. All spectra were obtained in the maxima visible in figure 9 , just above or below the hole. $(a, c, e)$ Grey solid line, $D=240 \mathrm{~nm}$; grey dashed-dotted line, $D=300 \mathrm{~nm}$; black dashed line, $D=350 \mathrm{~nm}$.

necessary to achieve a considerable $(75 \mathrm{~nm})$ shift in the peak position. This is in good agreement with the observation of clear peaks in the transmission spectrum of quasi-periodic structures [26]. In these structures, short-range order plays an important role as these structures lack long-range periodicity. 


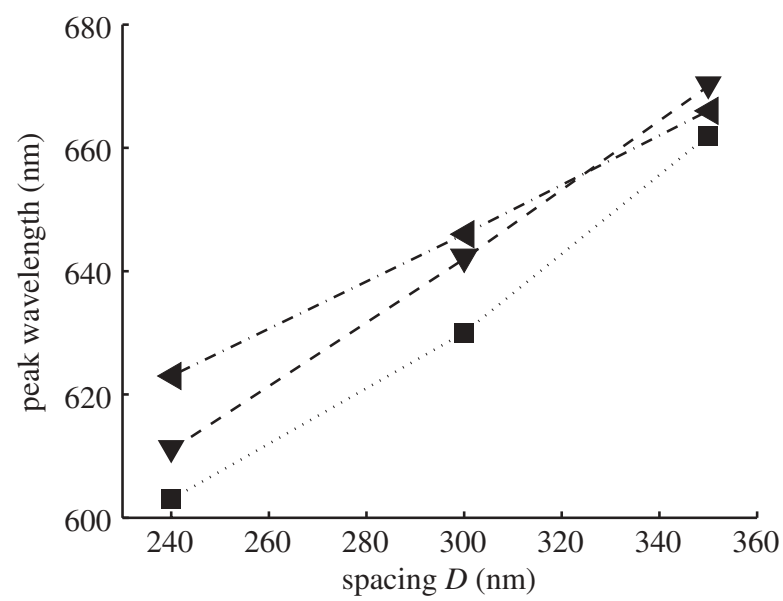

Figure 11. Positions of the peaks as a function of hole spacing $D$ for position 1 (left pointed triangles, top side of the top hole), position 2 (filled squares, lower side of the top hole) and position 3 (down pointed triangles, upper side of the middle hole).

We note here that the system under investigation has similarities with an antenna array, as was pointed out by Alaverdyan et al. [25]. The holes act as scatters with a well-defined phase difference, given by their distance. In our experiment, we can preferentially excite one of the elements of the antenna array. This resembles a transmitting antenna in which one scattering element is driven via an externally applied current and the other elements play a role for wavelength and directionality tuning of the antenna. Yet, the observed spectrum is the response of the entire structure rather than the response of a single hole.

\section{Conclusions}

Using a local excitation mechanism with a focused beam of electrons, we have measured the optical properties of rectangular subwavelength holes in the optical regime with a resolution far below the diffraction limit. Polarization-resolved measurements showed the polarization of the scattered light being predominantly oriented perpendicular to the hole edge. The spectrally resolved measurements on a large number of holes of different size and shape have revealed a clear dependence of the observed spectra on the dimension of the hole and the position at which the excitation was done. These results are an indication that the orthogonal waveguide modes present inside the rectangular hole play an important role in the local scattering processes. The mean wavelength observed at the edge of the hole follows the presence of modes in the waveguide and can thus be understood in terms of the cutoff wavelength of the holes. This is further supported by the observed spatial dependence of the polarization of the emission, which shows a preferential polarization perpendicular to the hole edge, as would be expected for modes in a metal waveguide. These results can be used as guidelines for the enhancement of molecular fluorescence near subwavelength holes for sensing applications. Linear arrangements of three identical holes show 
spectra that clearly differ from isolated spectra. These spectra can be tuned via the spacing between the holes, as well as the location of the exciting electron beam. This clearly shows that coupling between the holes plays an important role. In these multiple hole structures, the precise local excitation mechanism is expected to enable control over the directional emission of the antenna formed by the two-dimensional arrangement of holes.

The authors thank Matteo Burresi and Tobias Kampfrath for discussions and suggestions. The work is part of the research programme of the Stichting voor Fundamenteel Onderzoek der Materie (FOM), which is financially supported by the Nederlandse Organisatie voor Wetenschappelijk Onderzoek (NWO), and support by the NWO (VICI grant) is gratefully acknowledged. This research is also supported by NanoNed, a national nanotechnology programme coordinated by the Dutch Ministry of Economic Affairs (project number 6643).

\section{References}

1 Ebbesen, T. W., Lezec, H. J., Ghaemi, H. F., Thio, T. \& Wolff, P. A. 1998 Extraordinary optical transmission through sub-wavelength hole arrays. Nature 391, 667-669. (doi:10.1038/35570)

2 Garcia-Vidal, F. J., Martin-Moreno, L., Ebbesen, T. W. \& Kuipers, L. 2010 Light passing through subwavelength apertures. Rev. Mod. Phys. 82, 729-787. (doi:10.1103/RevModPhys. 82.729)

3 Bethe, H. 1944 Theory of diffraction by small holes. Phys. Rev. 66, 163-182. (doi:10.1103/ PhysRev.66.163)

4 Degiron, A., Lezec, H. J., Yamamoto, N. \& Ebbesen, T. W. 2004 Optical transmission properties of a single subwavelength aperture in a real metal. Opt. Commun. 239, 61-66. (doi:10.1016/j.optcom.2004.05.058)

5 Klein Koerkamp, K. J., Enoch, S., Segerink, F. B., van Hulst, N. F. \& Kuipers, L. 2004 Strong influence of hole shape on extraordinary transmission through periodic arrays of subwavelength holes. Phys. Rev. Lett. 92, 183901. (doi:10.1103/PhysRevLett.92.183901)

6 Gordon, R., Brolo, A. G., McKinnon, A., Rajora, A., Leathem, B. \& Kavanagh, K. L. 2004 Strong polarization in the optical transmission through elliptical nanohole arrays. Phys. Rev. Lett. 92, 037401. (doi:10.1103/PhysRevLett.92.037401)

7 Ropers, C., Park, D., Stibenz, G., Steinmeyer, G., Kim, J., Kim, D. \& Lienau, C. 2005 Femtosecond light transmission and subradiant damping in plasmonic crystals. Phys. Rev. Lett. 94, 113901. (doi:10.1103/PhysRevLett.94.113901)

8 Fischer, H., Nesci, A., Leveque, G. \& Martin, O. J. F. 2008 Characterization of the polarization sensitivity anisotropy of a near-field probe using phase measurements. J. Microsc. Oxford $\mathbf{2 3 0}$, 27-31. (doi:10.1111/j.1365-2818.2008.01954.x)

9 Adam, A. J. L., Brok, J. M., Seo, M. A., Ahn, K. J., Kim, D. S., Kang, J. H., Park, Q. H., Nagel, M. \& Planken, P. C. M. 2008 Advanced terahertz electric near-field measurements at sub-wavelength diameter metallic apertures: erratum. Opt. Express 16, 008054. (doi:10.1364/ OE.16.008054)

10 García de Abajo, F. J. 2010 Optical excitations in electron microscopy. Rev. Mod. Phys. 82, 209-275. (doi:10.1103/RevModPhys.82.209)

11 Kubo, A., Onda, K., Petek, H., Sun, Z., Jung, Y. \& Kim, H. 2005 Femtosecond imaging of surface plasmon dynamics in a nanostructured silver film. Nano Lett. 5, 1123-1127. (doi:10.1021/nl0506655)

12 Cinchetti, M., Gloskovskii, A., Nepjiko, S. A., Schönhense, G., Rochholz, H. \& Kreiter, M. 2005 Photoemission electron microscopy as a tool for the investigation of optical near fields. Phys. Rev. Lett. 95, 047601. (doi:10.1103/PhysRevLett.95.047601)

13 Nelayah, J. et al. 2007 Mapping surface plasmons on a single metallic nanoparticle. Nat. Phys. 3, 348-353. (doi:10.1038/nphys575)

14 Schaffer, B., Hohenester, U., Trugler, A. \& Hofer, F. 2009 High-resolution surface plasmon imaging of gold nanoparticles by energy-filtered transmission electron microscopy. Phys. Rev B 79, 041401. (doi:10.1103/PhysRevB.79.041401) 
15 Yamamoto, N., Araya, K. \& de Abajo, F. 2001 Photon emission from silver particles induced by a high-energy electron beam. Phys. Rev. B 64, 205419. (doi:10.1103/PhysRevB.64.205419)

16 Bashevoy, M., Jonsson, F., Krasavin, A., Zheludev, N., Chen, Y. \& Stockman, M. 2006 Generation of traveling surface plasmon waves by free-electron impact. Nano Lett. 6, 1113-1115. (doi:10.1021/nl060941v)

17 van Wijngaarden, J. T., Verhagen, E., Polman, A., Ross, C. E., Lezec, H. J. \& Atwater, H. A. 2006 Direct imaging of propagation and damping of near-resonance surface plasmon polaritons using cathodoluminescence spectroscopy. Appl. Phys. Lett. 88, 221111. (doi:10.1063/1.2208556)

18 Ginzburg, V. \& Tsytovich, V. 1990 Transition radiation and transition scattering. Bristol, UK: Adam Hilger.

19 Yamamoto, N. \& Suzuki, T. 2008 Conversion of surface plasmon polaritons to light by a surface step. Appl. Phys. Lett. 93, 093114. (doi:10.1063/1.2978248)

20 Kuttge, M., Vesseur, E. J. R., Koenderink, A. F., Lezec, H. J., Atwater, H. A., Garcia de Abajo, F. J. \& Polman, A. 2009 Local density of states, spectrum, and far-field interference of surface plasmon polaritons probed by cathodoluminescence. Phys. Rev. B 79, 113405. (doi:10.1103/PhysRevB.79.113405)

21 Popov, E., Bonod, N., Neviere, M., Rigneault, H., Lenne, P. \& Chaumet, P. 2005 Surface plasmon excitation on a single subwavelength hole in a metallic sheet. Appl. Opt. 44, 2332-2337. (doi:10.1364/AO.44.002332)

22 Baudrion, A.-L. et al. 2008 Coupling efficiency of light to surface plasmon polariton for single subwavelength holes in a gold film. Opt. Express 16, 3420-3429. (doi:10.1364/OE.16.003420)

23 Collin, S., Pardo, F. \& Pelouard, J.-L. 2007 Waveguiding in nanoscale metallic apertures. Opt. Express 15, 4310-4320. (doi:10.1364/OE.15.004310)

24 Hohenester, U., Ditlbacher, H. \& Krenn, J. R. 2009 Electron-energy-loss spectra of plasmonic nanoparticles. Phys. Rev. Lett. 103, 106801. (doi:10.1103/PhysRevLett.103.106801)

25 Alaverdyan, Y., Sepulveda, B., Eurenius, L., Olsson, E. \& Kall, M. 2007 Optical antennas based on coupled nanoholes in thin metal films. Nat. Phys. 3, 884-889. (doi:10.1038/nphys785)

26 Przybilla, F., Genet, C. \& Ebbesen, T. W. 2006 Enhanced transmission through penrose subwavelength hole arrays. Appl. Phys. Lett. 89, 121115. (doi:10.1063/1.2355450) 